\title{
Total magnitude, radius, colour indices, colour gradients and photometric type of galaxies ${ }^{\star, \star \star}$
}

\author{
Ph. Prugniel and Ph. Héraudeau \\ Centre de Recherches Astronomique de Lyon, CNRS, Observatoire de Lyon, F-69561 St-Genis-Laval Cedex, France
}

Received March 6; accepted July 21, 1997

\begin{abstract}
We present a catalogue of aperture photometry of galaxies, in $U B V R I$, assembled from three different origins: (i) an update of the catalogue of Buta et al. (1995) (ii) published photometric profiles and (iii) aperture photometry performed on CCD images.

We explored different sets of growth curves to fit these data: (i) The Sérsic law, (ii) The net of growth curves used for the preparation of the RC3 and (iii) A linear interpolation between the de Vaucouleurs $\left(r^{1 / 4}\right)$ and exponential laws. Finally we adopted the latter solution.

Fitting these growth curves, we derive (1) the total magnitude, (2) the effective radius, (3) the colour indices and (4) gradients and (5) the photometric type of 5169 galaxies. The photometric type is defined to statistically match the revised morphologic type and parametrizes the shape of the growth curve. It is coded from -9 , for very concentrated galaxies, to +10 , for diffuse galaxies.
\end{abstract}

Key words: galaxies: fundamental parameters galaxies: general — galaxies: photometry

\section{Introduction}

The magnitudes and diameters are two of the fundamental parameters defining the equilibrium status of galaxies. This is particularly well illustrated by the formalism of the Fundamental Plane of early type galaxies (Dressler et al. 1987; Djorgovski \& Davis 1987), where the total magnitude and effective radius scale the mass and the gravitational energy while the central velocity dispersion scales

Send offprint requests to: $\mathrm{Ph}$. Prugniel

(prugniel@obs.univ-lyon1.fr)

* Based in part on observations collected at the HauteProvence Observatory.

** Tables 1 to 4 are presented in electronic form only, and are available at the CDS via anonymous ftp to cdsarc.u-strasbg.fr (130.79.128.5) or via http://cdsweb.ustrasbg.fr/Abstract.html the kinetic energy, both being linked by the virial theorem. In addition, colours and colour gradients characterize the stellar populations (mean age and metallicity) and the amount and distribution of absorbing material. Also, the shape of the photometric profile (or of the growth curve) enters the scaling relations (Prugniel \& Simien 1997).

Since the beginning of the photometry of galaxies (Whitford 1936) the bulk of photometric data available has increased exponentially (Prugniel 1987). However, these data are inhomogeneous both in their quality and form: They are based on photoelectric, photographic or CCD observations, and they are presented as centered aperture photometry through circular or elliptical apertures or as photometric profiles. The largest effort for deriving the fundamental parameters on a whole-sky homogeneous form was part of the production of the Third Reference Catalogue (RC3) (de Vaucouleurs et al. 1991; Buta et al. 1995; Buta \& Williams 1995). The principle was to use a catalogue of photoelectric photometry (an update of the catalogues of aperture photometry by Longo \& de Vaucouleurs 1983, 1985, 1988) to fit curves of growth and to derive magnitudes, diameters and colours. Each individual original reference from these catalogue was analysed, corrected for systematic effects and affected a weight.

In order to take into account the continuously growing amount of of photometric data on galaxies, we have written a non-interactive growth curve fitting algorithm to derive magnitudes, radii, colour and colour gradients and photometric type (ie. shape of the growth curve).

Our photometric database consists in: (1) an update of the Longo \& de Vaucouleurs catalogues of photoelectric aperture photometry, (2) the photographic aperture photometry listed in the computerized version of the ESO-LV catalogue (Lauberts \& Valentjin 1989, hereafter ESO-LV, (3) aperture photometry derived from published photometric profiles, mostly from CCD observations (in particular the $I$-band photometry by Mathewson et al. 1992), and (4) CCD photometry obtained at the $1.2 \mathrm{~m}$ telescope of the Observatoire de Haute-Provence. Using this database 
we already derived the photometric parameters used in Prugniel et al. (1993) and Prugniel \& Simien (1994-1997).

In this paper we present our database (Sect. 2) and the fitting method and homogenisation (Sect. 3). The shape of growth curves is analysed in Sect. 4 and the derived parameters and comparison with $\mathrm{RC} 3$ are presented in Sect. 5. We discuss the physical meaning of the photometric type in Sect. 6.

\section{The database of aperture photometry}

The aperture photometry catalogues published by Longo and de Vaucouleurs (1983, 1985, de Vaucouleurs \& Longo $1988)$ gather photoelectric aperture photometry in $U, B$, $V, R$ and $I$ and are almost complete until 1985 in $U, B$ and $V$, and 1988 in $R$ and $I$. We completed these catalogues with more recently published aperture photometry (see references in Table 2) and we merged it with the catalogue used in Buta et al. (1995). We did not consider here the infrared bandpasses $(J, H, K, \ldots)$ because the corresponding measurements are based on a different technology and because their availability is still restricted compared to the visible photometry. Beside the photoelectric aperture photometry, we used the aperture photometry given in the ESO-LV catalogue (we considered only the $B$-band) and we selected 2-dimensional CCD photometry from the literature. For the star-resolved dwarf spheroidal companions of the Galaxy, we used published star-count profiles.

We selected from the litterature the 2-dimensional CCD photometry after two criteria: (1) The published data are suited to our purpose (this excludes, in particular, the profiles for which the central part, saturated or presumably affected by the seeing, is not presented). (2) The data are available in electronic form from the authors or from CDS or in the form of printed tables, which we re-digitized (we did not use profiles published as graphics). Finally, our database does not pretend to be complete: digitizing all the published table would require a huge amount of work.

With a few exceptions (e.g. Djorgovski 1985) the authors of surface photometry do not give integrated magnitude within centered circular apertures but within ellipses of varying position angle and ellipticity. Hence, we used the ellipses parameters to re-construct images on which we simulated aperture photometry. The set of simulated aperture diameters was chosen to match the published sampling along the minor axis. When the information was given by the authors, we retained only the apertures of diameter larger than 3 times the FWHM seeing and with an internal error on the integrated magnitude smaller than 0.05. When the information could not be derived from the description provided by the authors, we statistically determined it from the distribution of the residuals from the fit (i.e. the residuals from the growth curves depart toward larger magnitude for apertures affected by the seeing, we retained only the aperture large enough for that effect beeing smaller than 0.05 mag in average).

Excluding from the sample the galaxies having only photometry from the ESO-LV, our database contains aperture photometry on a total of 7744 galaxies from 445 references. Photoelectric photometry is available for 6129 galaxies, and surface photometry for 2774 galaxies.

The galaxy identifiers were adopted by descending the hierarchy: (a) NGC name, (b) IC, (c) UGC or ESO, (d) PGC (Principal Galaxies Catalogue, Paturel et al. 1992), and (f) LEDA internal identifier (also acronymed PGC as adopted in SIMBAD). We made use of the LEDA database (operated at Observatoire de Lyon) and SIMBAD (operated by Observatoire de Strasbourg) for doing the crossidentification. Finally, 7472 galaxies could be folded into this hierarchy of identifiers. The remaining 272 objects (4\%) are identified by their coordinates (identifier A) and could not be identified with any galaxy in LEDA, whether because the coordinates are not known with a sufficient precision (possibly leading to an ambiguous identification) or because the galaxy is absent from LEDA.

Table 1, available only in electronic form, presents the aperture catalogue.

\section{Fitting method and homogenisation of the data}

The growth curve fitting method has been initiated and refined in the successive versions of Reference Catalogues (de Vaucouleurs \& de Vaucouleurs, $1964=\mathrm{RC} 1$, de Vaucouleurs et al. $1976=\mathrm{RC} 2$; de Vaucouleurs et al. 1991 RC3). In this line of work, the growth curves (i.e. fractional flux vs normalized aperture) are chosen dependent on the morphological type of the galaxy, i.e. a different growth curve is adopted for each morphological type. The net of growth curves was adopted by averaging the growth curves obtained for small sets of template galaxies of each morphological type.

\subsection{Photometric type}

In the RC3, when the photometric data were numerous and good enough the fit was performed with the different growth curves and the best fit adopted for the determination of the effective radius and total magnitude. Hence, this determined a "photometric" type (see Buta et al. 1995), by definition correlated with the morphological type (because the growth curves were constructed by averaging the observed growth curves of template galaxies sampling the range of morphological types). However, the correlation was not good enough for the photometric type being considered as a measurement of the morphological type.

Because the present database includes a large amount of aperture photometry derived from CCD observations (characterized by a very high internal consistency and well 
sampled growth curves), it will be possible to generalize the determination of the photometric type.

\subsection{Fitting of growth curves}

The growth curves are expressed as functions of the photometric type $T_{\text {phot }}$, and of the normalised radius of the circular aperture $x=A / A_{\mathrm{e}}$ where $A$ is the aperture diameter and, $A_{\mathrm{e}}$ the diameter of the effective aperture, in tenths of an arcmin. They are defined as:

$g\left(T_{\mathrm{phot}}, x\right)=m_{\infty}-m(x)$

where $m(x)$ is the integrated magnitude within the aperture $x$, and, $m_{\infty}=m(\infty)$ is the asymptotic (or total) magnitude.

The adopted nets of growth curves are described in Sect. 4. The photometric type is defined to match the morphological type coded as in RC2 by setting two conditions: (1) $g(-5, x)$ corresponds to the de Vaucouleurs law and $(2) g(10, x)$ to the exponential law.

Considering a set of measured magnitudes, $m_{i}$, the fit, by varying $A_{\mathrm{e}}$ and $T_{\text {phot }}$, consists in minimizing the quantity:

$\delta^{2}=\sum\left(w_{i} \delta_{i}\right)^{2}$

where $\delta_{i}$, the residual (in mag) for the measurement $i$, is:

$\delta_{i}=\left(m_{i}(r)-g\left(T_{\mathrm{phot}}, x\right)-c_{i}-m_{\infty}\right)$

$m_{\infty}$, being determined from:

$\sum\left(w_{i} \delta_{i}\right)=0$.

The correction term $c_{i}$ is:

$c_{i}=c_{c}(i)+c_{r 0}(i)+c_{r c}(i)$.

The different components of $c_{i}$ are defined as follows:

$-c_{c}(i)$ is the reduction of the magnitude measured in the colour $c$ into the $B$-band. It is based on a linear fit of the colour indices vs. $\log (A)$ relations and is detailed in Sect. 3.2

$-c_{r 0}(i)$ is a zero-point correction applied to all the measurements (converted into $B$ ) of the considered galaxy from reference $r$ (see details Sect. 3.4)

$-c_{r c}(i)$ is a systematic correction applied to all the measurements of reference $r$ in the colour $c$. The determination of these corrections is detailed in Sect. 3.5

and, in Eq. (3), $w_{i}$, the weight attached to measurement $i$, is:

$w_{i}=w_{c}(i) w_{r 0}(i) w_{r g}(i) w_{r \mathrm{~s}}(i) w_{\mathrm{d}}(i) w_{\mathrm{a}}(i)$

where,

$-w_{c}(i)$ is the weight associated with the uncertainty of the "colour reduction", i.e. the computation of $c_{c}(i)$. $-w_{r 0}(i)$ accounts for the precision of the zero-point determination of reference $r$.

- $w_{r g}(i)$ is a weight globally affected to all the measurements (in all the colours) from reference $r$.

$-w_{r \mathrm{~s}}(i)$ accounts for the density of the sampling of reference $r$.

$-w_{\mathrm{d}}(i)$ is a clipping function aimed at rejecting the discrepant measurements.

$-w_{\mathrm{a}}(i)$ reduces the weight of the small apertures.

For each galaxy, the fit was performed using a downhill method, starting from guesses of $\log \left(A_{\mathrm{e}}\right)$ and $T_{\text {phot }}$. The guess for $\log \left(A_{\mathrm{e}}\right)$ was an average of the measured apertures, and for $T_{\text {phot }}$ it was the morphological type (hereafter $T_{\text {mor }}$ ) taken from LEDA. When $T_{\text {mor }}$ was not available in LEDA, we assumed $T_{\text {phot }}=-5$ for galaxies noted as "Compact" and $T_{\text {phot }}=+5$ when classified as "Diffuse". For 300 remaining galaxies without any structure indication reported in LEDA, we arbitrarily assumed initial $T_{\text {phot }}=0$, but determined the photometric parameters only if the aperture data were sufficient to fit $T_{\text {phot }}$. The morphological type of a galaxy in LEDA is the weighted average of the different estimations available in the database. According to Naim et al. (1995), the dispersion around such determination is typically 1.8. Because the growth curves are monotonic, the choice of the starting values only changes the rapidity of the convergence of the fit.

We fitted the photometric type $\left(T_{\text {phot }}\right)$ if at least 10 apertures were available and if the range in $\log (A)$ exceeded 0.7 . Otherwise, we adopted the morphological type $T_{\text {mor }}$ for the determination of the other parameters, but these galaxies were not included in the figures or in the analysis of the residuals. The fitting procedure is automatic. When the routine does not converge to stable values we only used the upper part of the growth curve to get the total magnitude. At each step $c_{c}(i), c_{r 0}(i), w_{c}(i), w_{\mathrm{d}}(i)$ and $w_{\mathrm{a}}(i)$ were recomputed, and $w_{r 0}(i)$ was progressively rised to 1 as $c_{r 0}$ was refined.

We initially started from equal weights $w_{r g}$ and $w_{r 0}$, and no $c_{r c}$ corrections for all the references, and we computed these weights and corrections by analysing the residuals from the fit for all the galaxies of our sample. The determinations of the different parameters, correction terms and their weights converged to stable values after about 10 iterations. As new references were incorporated in the database, they were given initial low weights afterwards set to their stable values.

\subsection{Determination of the colour indices $\left(c_{c}\right)$ and associ- ated weights $\left(w_{c}\right)$}

The growth curve fitting is done in the $B$-band, and the measurements done in other bandpasses are converted to 


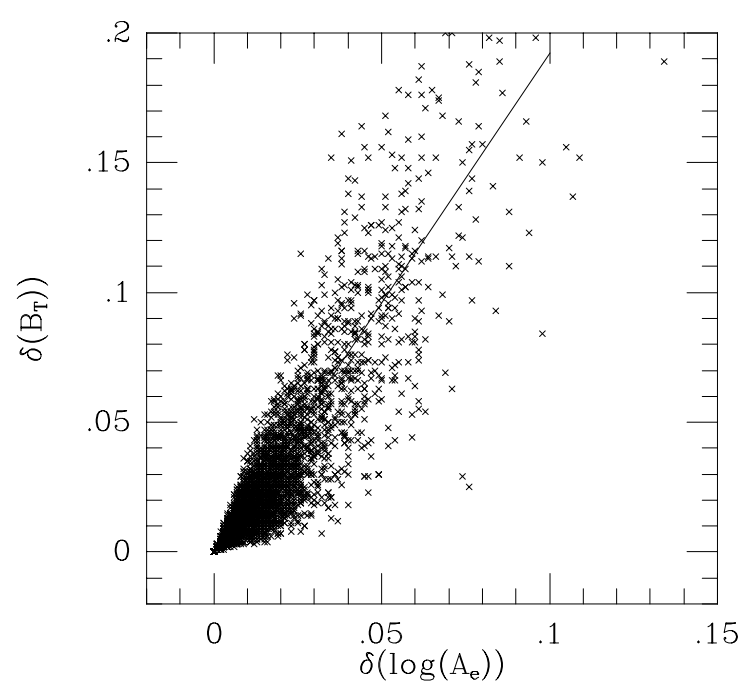

Fig. 1. Correlation of the internal errors on $\log \left(A_{\mathrm{e}}\right)$ and $B_{\mathrm{T}}$. In abscissae: The error on $\log \left(A_{\mathrm{e}}\right)$ (expressed in tenth of arcmin). In ordinates: The error on $B_{\mathrm{T}}$ (in mag). The straight line is the regression $\delta\left(B_{\mathrm{T}}\right)=1.9 \delta\left(\log \left(A_{\mathrm{e}}\right)\right)$

the $B$-band by fitting a linear relation between the observed colour indices, $c_{o c}$, and $\log (r)$ :

$c_{o c}(i)=\alpha_{c}+\beta_{c} \log \left(r_{i}\right)$

where $\alpha_{c}$ and $\beta_{c}$ are the two parameters fitted for the colour $c$ by minimizing:

$$
\begin{array}{r}
\sum\left(w_{r \mathrm{~g}}(i) w_{r 0}(i) w_{r \mathrm{~s}}(i) w_{\mathrm{d}}(i) w_{\mathrm{a}}(i)\right. \\
\left(\alpha_{c}+\beta_{c} \log \left(r_{i}\right)-c_{o c}(i)\right)^{2} .
\end{array}
$$

The colour index entering Eq. (5) is thus:

$c_{c}(i)=\alpha_{c}+\beta_{c} \log \left(r_{i}\right)$

This fit provides with the colours reduced at the effective aperture and the colour gradients.

When the direct calibration in the $B$-band was not possible, for example, if a galaxy was only observed in the $R$-band, the conversion toward the $B$-band was done using the mean colour index corresponding to the adopted value of $T_{\text {phot }}$. These were determined a posteriori, see Sect. 5 .

The reduction to the $B$-band was recomputed at each iteration of the growth curve fitting to account for the detection of discrepant measurements (through the clipping function $w_{\mathrm{d}}$ ).

\subsection{Zero-point correction $\left(c_{r 0}\right)$ and weight $\left(w_{r 0}\right)$}

An important characteristic of our database is the mixing of photoelectric and CCD photometry. While the latter has a very high internal consistency $(\approx 0.01 \mathrm{mag}$ against $\approx 0.04$ mag for photoelectric photometry) its external precision (error on the zero-point) is not better, and often worse than that of the former.

To account for this characteristic, we computed a correction, $c_{r 0}$ of the zero-point of each reference during the fit of the growth curve.

$c_{r 0}$ is evaluated as the difference between the mean residual for reference $r$ and for all the others. This comparison is restricted to the range in $\log (x)$ where measurements from more than one reference are available. The correction is adopted if it is computed from at least 3 measurements and if it is decided statistically significant.

The weight $w_{r 0}$ is determined from the distribution of the $c_{r 0}$ for all the sample.

The adopted $w_{r 0}$ are listed in Table 3.

\subsection{Corrections $\left(c_{r c}\right)$ and weights $\left(w_{r \mathrm{~g}}\right)$ associated with each reference}

We analysed the $\delta_{i}$ for all the sample, searching for systematic effects associated with the reference.

We determined colour-dependent corrections to individual references, $c_{r c}$, whenever a significant systematic residual was found.

The weights, $w_{r g}$, are computed from the rms dispersion of the residuals for reference $r$.

These corrections and weights were computed after several iterations over the whole sample, until stability was reached. The corresponding $w_{r \mathrm{~g}}$ and $c_{r c}$ are listed in Table 3.

\subsection{Sampling density weight $\left(w_{r \mathrm{~s}}\right)$}

A major source of uncertainty in the photometric measurements results from the error in determining the sky background to be subtracted. In general, each photoelectric measurement of an object is accompanied with a sky measurement, hence, even in a series of observations (multi-aperture), all the observed magnitudes are independent. At the contrary, the surface photometry proceeds to a single (and likely more precise) sky determination. Consequently, the magnitudes measured through the different apertures are not completely independent. Hence, a finer sampling of the aperture range would unduely increase the effective weight of the corresponding reference. To tackle this effect, the weight is decreased by the factor $w_{r \mathrm{~s}}=15 / \bar{m}$, where $\bar{m}$ is the average number of aperture measured for each galaxy in reference $r$, if $\bar{m}>15$.

\subsection{Clipping of the discrepant measurements $\left(w_{\mathrm{d}}\right)$}

After each downhill step of the growth curve fit, the residuals are searched for the detection of discrepant measurements. Data farther than $3 \sigma$ are clipped, i.e. $w_{\mathrm{d}}(i)=0$, and to avoid instability, $w_{\mathrm{d}}$ is reduced for data in the range $2-3 \sigma$. For other measurements, $w_{\mathrm{d}}=1$. 


\subsection{Aperture dependent weight $\left(w_{\mathrm{a}}\right)$}

On the one hand, the small apertures are affected by seeing effect and, for the photoelectric photometry, by centering errors. On the other hand, the large apertures are affected by sky subtraction errors. To take into account these two effects, the analysis of the residuals for the whole sample lead us to compute a weight $w_{\mathrm{a}}(\log A)$ from the variation of the rms residuals with $\log A$.

\subsection{The RC3 weighting function, and special corrections}

A different system of weighting was adopted for the preparation of the RC3 (Buta et al. 1995). It is function of the reference but also of the telescope aperture and measured magnitude. We did not perform a detailed comparison with our weighting function, but qualitatively, the zero-point corrections agree and both approaches show the highest quality of the most recent observations.

The residuals for each references have been analysed separately. In a couple of cases, this lead to corrections which are not included in Eq. (5).

For example, the reference MIC - 82 (see Table 2) is better corrected by subtracting 0.03 to $\log (A)$ than changing the magnitude zero-point. It may be due to an error on the telescope scale, or to the use of an octogonal diaphram. Apertures were also modified in references HOD - 63 and WEG - 79. For the reference DJO - 85 (CCD in $R$ ), we individually recalibrated the zero-point when it was possible.

\subsection{Error estimates}

The determination of the errors is derived from the distribution of the residuals for a considered galaxy:

$e_{1}=\sqrt{\frac{\delta^{2}}{\sum w_{i}} \frac{1}{N-f}}$

where $N$ is the number of observations and $f$ the number degrees of freedom: $f=3+h$, (if $T_{\text {phot }}$ was fitted) where $h$ is the number of references corrected for zero-point.

Hence, the errors on the three parameters were determined by estimating the variations in $m_{\infty}, \log \left(A_{\mathrm{e}}\right)$ and $T_{\text {phot }}$ associated with $e_{1}$, respectively $e_{1}, e_{2}$ and $e_{3}$.

Because of the non-linearity of the fit, the errors on each parameter are not independent, i.e. the error box is not an ellipsoid with axes parallels to the axes of the parameter space. In particular, the correlation of the errors on $m_{\infty}$ and $\log \left(A_{\mathrm{e}}\right)$ is well documented, see e.g. Hamabe $\&$ Kormendy (1987). Thus, we computed the errors, $e_{4}$ and $e_{5}$, on $m_{\infty}$ resulting from $e_{2}$ and $e_{3}$.

The total error on $m_{\infty}$ is:

$\delta\left(m_{\infty}\right)=\sqrt{\left(e_{1}^{2}+e_{4}^{2}+e_{5}^{2}\right)}$.

The correlation between the internal errors on $m_{\infty}$ and on $\log \left(A_{\mathrm{e}}\right)$ is shown in Fig. 1 .

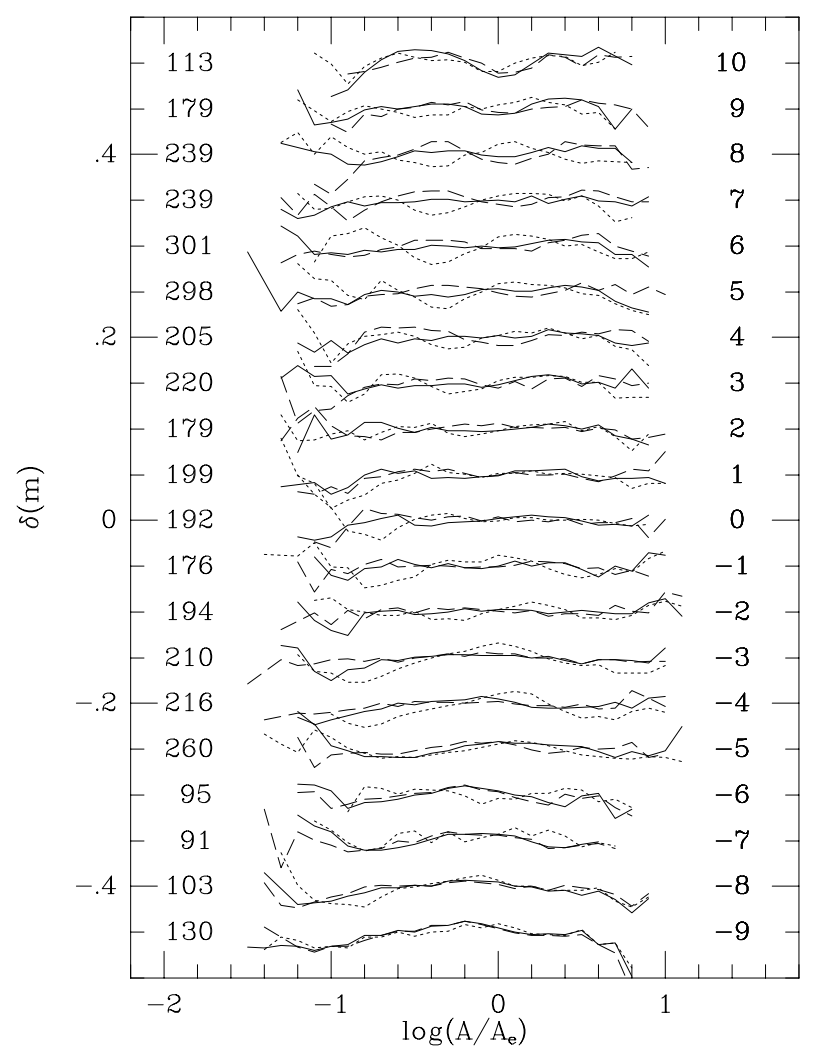

Fig. 2. Mean residuals from growth curves. Full line: INTERP, Dashed line: Sérsic, Dotted: RC3. Each curve represents the mean residuals (ordinate in mag; $\delta(m)=$ Observed Computed) vs. the normalized aperture (abscissa) for the galaxies with $T_{0}-0.5<T_{\text {phot }}<T_{0}+0.5$ where the photometric type $T_{0}$, labelled on the right side, runs from -9 to 10. The number of galaxies used to built each curve for the INTERP fit is labelled on the left side (for the other fits, the numbers are comparable). Each curve is shifted by a zero point $\delta\left(m_{0}\right)=0.05 T_{\text {phot }}$

\section{The shape of growth curves}

In the Reference Catalogue of Brigth Galaxies (RC1, de Vaucouleurs \& de Vaucouleurs 1964) the asymptotic magnitudes were derived from two parabolic growth curves, one for the early type galaxies and one for late types. Then in the $\mathrm{RC} 2$, the shape of the growth curves was determined for each revised morphological type using photographic surface photometry (de Vaucouleurs 1977). For each type, 4 to 12 galaxies were available. The set of growth curves used in the RC3 results from the evolution of the curves used in the $\mathrm{RC} 2$ : the mean residuals from growth curves for each morphological type were used to compute differential corrections.

In the present work, surface photometry is available for 2774 galaxies. Hence we decided to reconsider the determination of the shape of the growth curves. 


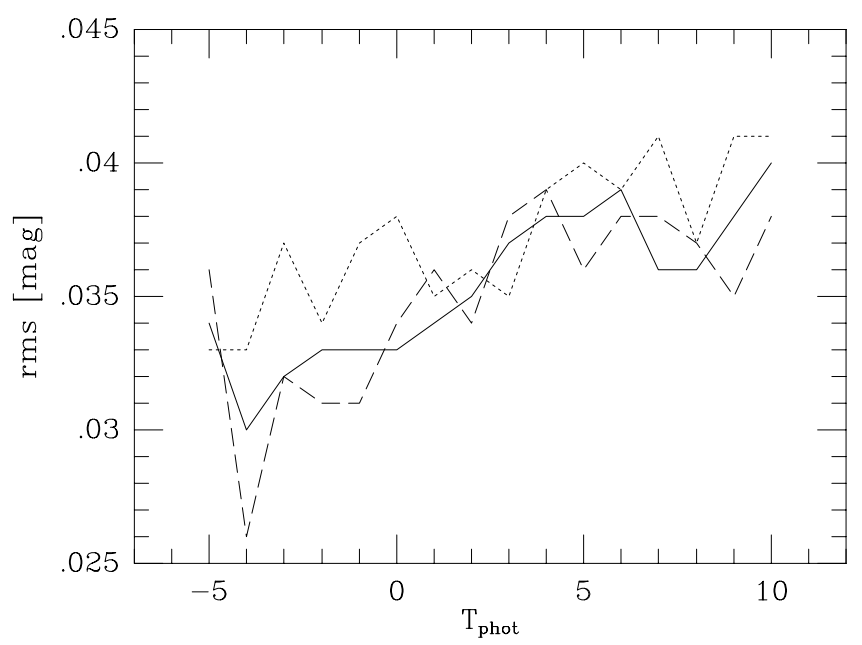

Fig. 3. Rms residuals from the growth curves fit vs. $T_{\text {phot }}$. Dashed line: Sérsic, Dotted: RC3, Continuous: INTERP

A major difference with RC3 is that we are determining the shape of the growth curves independently of the morphological type, hence, we call the parameter coding this shape the photometric type. We have chosen to write the photometric type in a scale comparable to the revised morphological type by setting the conditions:

$-T_{\text {phot }}=10$ for the dwarf galaxies with exponential profile.

$-T_{\text {phot }}=-5$ for the elliptical galaxies with de Vaucouleurs profile.

This scale extends toward $T_{\text {phot }}<-5$, reflecting the existence of more concentrated objects, as discussed in Caon et al. (1990).

\subsection{Determination of the growth curve}

We performed the fits with the three following sets of growth curves. Each of them spanning the full range of growth curves shapes, between exponential laws and structures even more concentrated than de Vaucouleurs law:

- Sérsic growth curves: The Sérsic growth curves were used in Prugniel \& Simien (1997) and proved to efficiently characterize the early-type galaxies. Indeed, the derived Sérsic exponent, $n$, was found to correlate with other galaxy parameters, and in particular with the residuals from the Fundamental Plane.

In the present case, we converted the Sérsic exponent into the photometric type with:

$T_{\text {phot }}=15-5 n$.

This relation returns the de Vaucouleurs law for $T_{\text {phot }}=-5$ and the exponential law for $T_{\text {phot }}=10$.

The comparison between the fitted $T_{\text {phot }}$ and the morphological type (see Sect. 5) did not justify any sophistication of this relation.

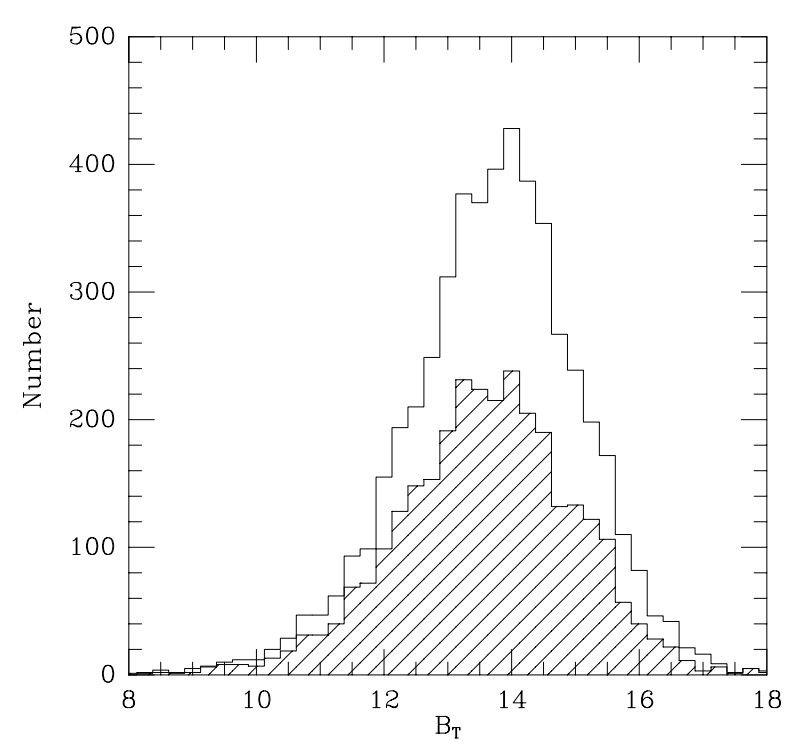

Fig. 4. Histogram of total $B$-magnitude, $B_{\mathrm{T}}$, for the whole sample and for galaxies with a quality $Q \leq 2$ (hatched)

- RC3 growth curves:

The RC3 growth curves (RC3, Vol. 1, p. 28) were derived by averaging, for each morphological type, the growth curves of template individual galaxies with available surface photometry.

For consistency with our other sets of growth curves, we extrapolated the RC3 set toward values of $T_{\text {phot }}<$ -5 by Sérsic growth curves.

- de Vaucouleurs - exponential interpolation (hereafter INTERP):

We finally used a linear combination between the de Vaucouleurs $g_{0}$ and exponential $g_{1}$ laws:

$$
\begin{array}{r}
g\left(T_{\text {phot }}, x\right)=\left(2 / 3-T_{\text {phot }} / 15\right) g_{0}\left(T_{\text {phot }}, x\right)+ \\
\left(1 / 3+T_{\text {phot }} / 15\right) g_{1}\left(T_{\text {phot }}, x\right) .
\end{array}
$$

As in the Sérsic case, no further refinement was needed.

\subsection{Adopted growth curves}

The residuals from the growth curve fitting are displayed in Fig. 2 for the three cases above. For computing these mean residuals we only selected the galaxies for which the fit was of the best quality ( $Q \leq 2$, see next section).

We note that the RC3 growth curves for late types were not modified since $\mathrm{RC} 2$, and are probably out of date, in particular considering the significant contribution to the determination of the growth curves constituted by the inclusion of the photometry of 1355 late type galaxies from Mathewson et al. (1992). (Unfortunately, this latter photometry is restricted to the $I$ band. Thus, color gradients had to be assumed or fitted on other data). This may partly explain the systematical wawy pattern apparent in Fig. 2. 


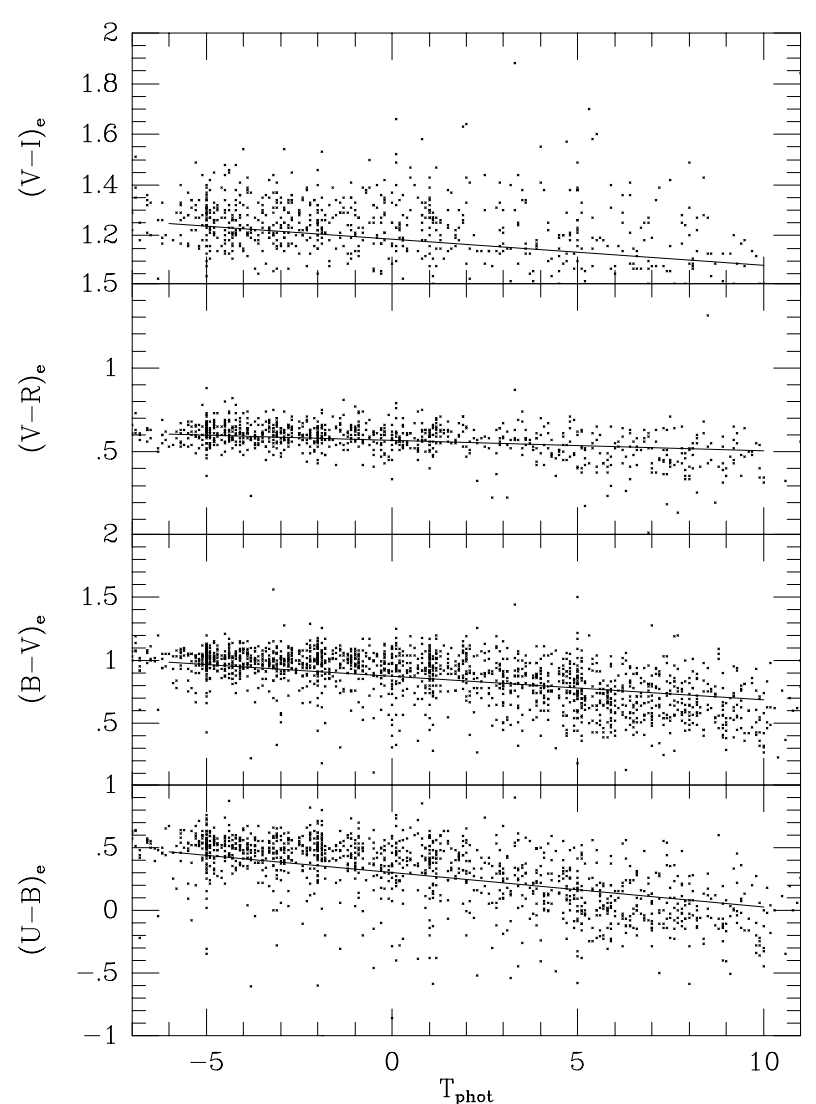

Fig. 5. Relation between the effective colour indices and the photometric type. The continuous lines are the least square fitted solution adopted for reducing the data to the $B$-band when no direct calibration were available (See Sect. 5.2)

Figure 3 shows the rms dispersion about the growth curves as a function of the photometric type for the three sets. Both Sérsic and INTERP growth curves are comparable, while RC3 is significantly worse.

Finally, we adopted INTERP rather than Sérsic growth curves, because, in the course on this work, analysing a restricted sample of late type galaxies, we noted a systematic pattern in the residuals of the latter, absent from the former.

\section{Derived parameters and comparison with RC3}

We ran the growth curve fitting program only for the galaxies cross-identified in LEDA. Out of them the photometric type (see Sect. 4) was determined for 3262 galaxies. Figure 4 shows the histogram of $B_{\mathrm{T}}$ for the whole sample and for galaxies of quality $Q \leq 2$.

\subsection{Derived parameters}

The derived parameters are summarized in Table 4 for the 5169 galaxies for which a growth curve could be fitted ( 2229 were rejected because only one aperture or two apertures from different references were available).

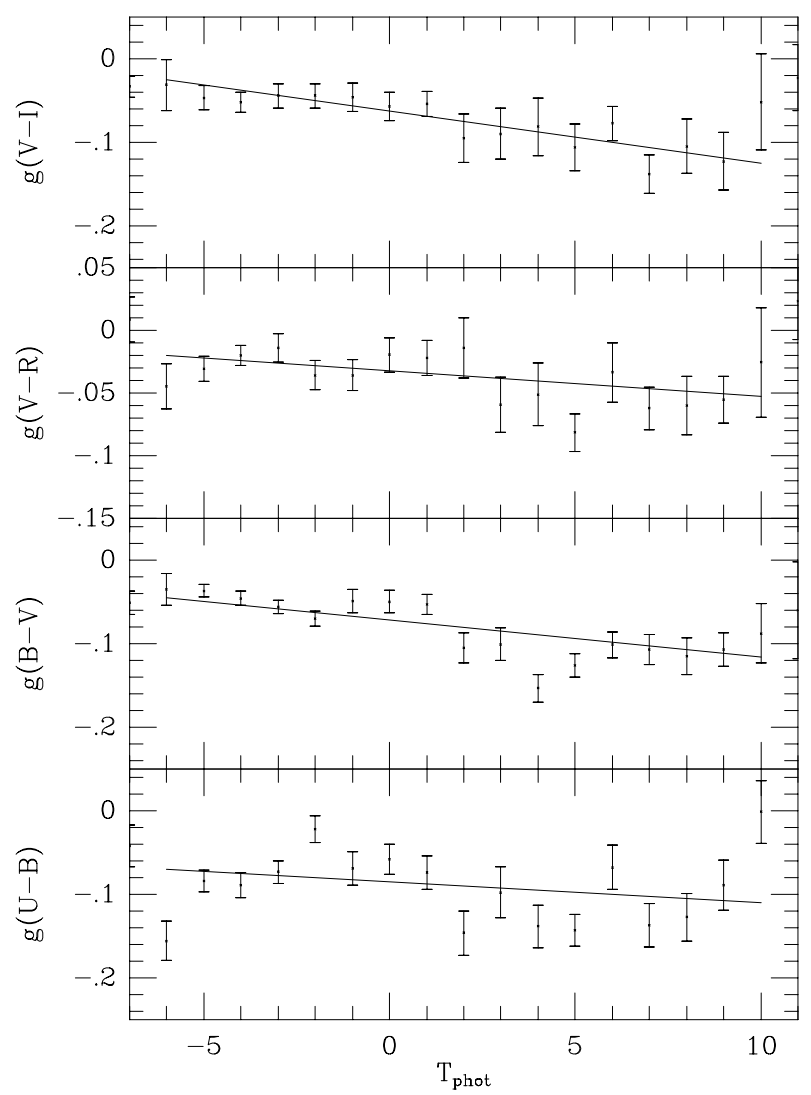

Fig. 6. Relation between the colour gradients (in ordinates) and the photometric type. The points are the average gradients (in $\mathrm{mag} / \log (0.1 \mathrm{arcmin})$ computed in bins of width $\delta\left(t_{\text {phot }}\right)=1$. The error bars are the uncertainty on this mean gradient, i.e., rms $/(N-1)^{1 / 2}$, where $\mathrm{rms}$ is the dispersion within a given box and $N$ the number of galaxies in this box. Typically, $\mathrm{rms} \approx 0.1$ and $N \approx 100$. The continuous lines are the least square fitted solution adopted for reducing the data to the $B$-band when no direct determination of the colour gradients were possible

\subsection{Comparisons with RC3}

Figure 7 compares the total $B$ magnitudes from RC3 and this study, respectively $B_{\mathrm{T}}(\mathrm{RC} 3)$ and $B_{\mathrm{T}}(\mathrm{PH})$, and Table 7 summarizes the results of the comparison of different derived parameters. The comparisons were restricted to the galaxies with $Q \leq 2$ and with an error on $\mathrm{BT}(\mathrm{RC} 3)$ smaller than 0.4 .

The systematic differences are significant. In average, our determinations are $\approx 0.05 \mathrm{mag}$ more luminous, and $\approx 0.15 \mathrm{mag} / \operatorname{arcsec}^{2}$ less bright than those in RC3. The differences in colours are consequences of those on $B_{\mathrm{T}}$ and $\mu_{\mathrm{e}}$, taking the colour gradients into consideration. These differences are due to the choice of the set of growth curve, as when using the RC3 set of growth curves our determinations are consistent with $\mathrm{RC} 3$ to within $0.01 \mathrm{mag}$ on $B_{\mathrm{T}}$. 


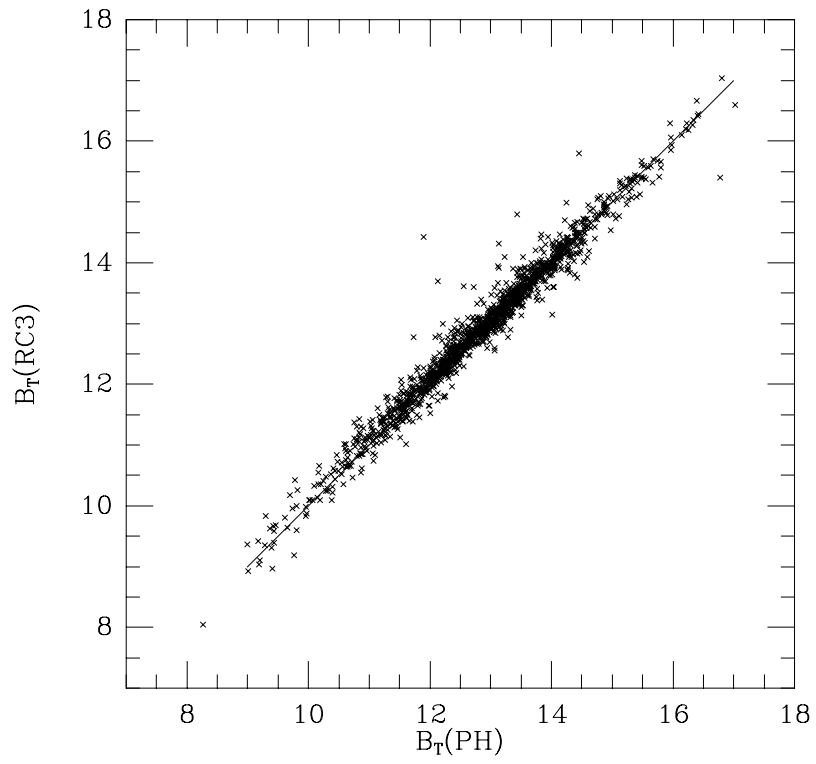

Fig. 7. Comparison between total magnitudes from RC3, $B_{\mathrm{T}(\mathrm{RC} 3)}$ (in ordinate), and from this study, $B_{\mathrm{T}(\mathrm{PH})}$ (abscissa). The comparison is done for 1403 galaxies having the quality flag: $Q \leq 2$. The straight line corresponds to $B_{\mathrm{T}(\mathrm{RC} 3)}=B_{\mathrm{T}(\mathrm{PH})}$

Table 5. Coefficients of the linear regression between colour indices $\left(u_{\mathrm{c}}, v_{\mathrm{c}}\right)$ and colour gradients $\left(u_{\mathrm{g}}, v_{\mathrm{g}}\right)$ with $T_{\text {Phot }}$. Coefficients $u$ are the ordinates for $T_{\mathrm{phot}=0}$ and $v$ the slopes. (See Figs. 5 and 6)

\begin{tabular}{|l|r|r|r|r|}
\hline Colour & $u_{\mathrm{c}}$ & $v_{\mathrm{c}}$ & $u_{\mathrm{g}}$ & $v_{\mathrm{g}}$ \\
\hline$U-B$ & 0.300 & -0.027 & -0.084 & -0.002 \\
$B-V$ & 0.870 & -0.019 & -0.071 & -0.004 \\
$V-R$ & 0.570 & -0.006 & -0.030 & -0.002 \\
$V-I$ & 1.179 & -0.011 & -0.059 & -0.006 \\
\hline
\end{tabular}

The few outliers apparent in Fig. 7 are galaxies for which the RC3 fit was based on very few apertures. They are: IC 5273 and NGC 46034679508553987649

Figure 8 shows the error correlation between $B_{\mathrm{T}}$ and $\log \left(A_{\mathrm{e}}\right)$, the line is the correlation observed for internal errors (see Fig. 1). As already noticed by, e.g., Hamabe \& Kormendy (1987), this correlation is important. It may significantly biases physical relations, in particular those used as distance indicator. As illustrated by the comparisons with RC3, the external errors on $B_{\mathrm{T}}$ and $\mu_{\mathrm{e}}$ are much larger than those derived from the formal errors on our fitting parameters. If we a priori suppose that the errors on both determinations are comparable, the external errors are $0.15 \mathrm{mag}$ on $B_{\mathrm{T}}$ and $0.30 \mathrm{mag} / \operatorname{arcsec}^{2}$ on $\mu_{\mathrm{e}}$. The comparisons between the results obtained with the three sets of growth curves (INTERP, Sérsic, and RC3) give comparable rms differences. This shows that the intrinsic deviations from any set of growth curves for individual galaxies is the dominant source of error.
Table 6. Distribution of the galaxies with the quality $(Q)$

\begin{tabular}{|r|r|}
\hline$Q$ & Nb of gal \\
\hline 0 & 2191 \\
1 & 751 \\
2 & 2253 \\
3 & 751 \\
4 & 823 \\
5 & 152 \\
6 & 371 \\
\hline
\end{tabular}

Table 7. Comparisons with RC3. $N$ is the number of galaxies used for the comparison $\left(Q \leq 2\right.$ and error on $\left.B_{\mathrm{T}(\mathrm{RC} 3)} \leq 0.4\right)$. The units mag $/ \operatorname{arcsec}^{2}$ for $\mu_{\mathrm{e}}$ and mag for the other quantities

\begin{tabular}{|r|r|r|c|}
\hline Quantity & $N$ & $\begin{array}{r}\text { Mean difference } \\
\text { PH - RC3 }\end{array}$ & rms \\
\hline$B_{\mathrm{T}}$ & 1648 & -0.057 & 0.242 \\
$\mu_{\mathrm{e}}$ & 1509 & 0.159 & 0.485 \\
$(U-B)_{\mathrm{e}}$ & 1172 & -0.006 & 0.083 \\
$(B-V)_{\mathrm{e}}$ & 1501 & 0.003 & 0.045 \\
$(V-R)_{\mathrm{e}}$ & 376 & 0.009 & 0.050 \\
$(V-I)_{\mathrm{e}}$ & 374 & 0.007 & 0.090 \\
\hline
\end{tabular}

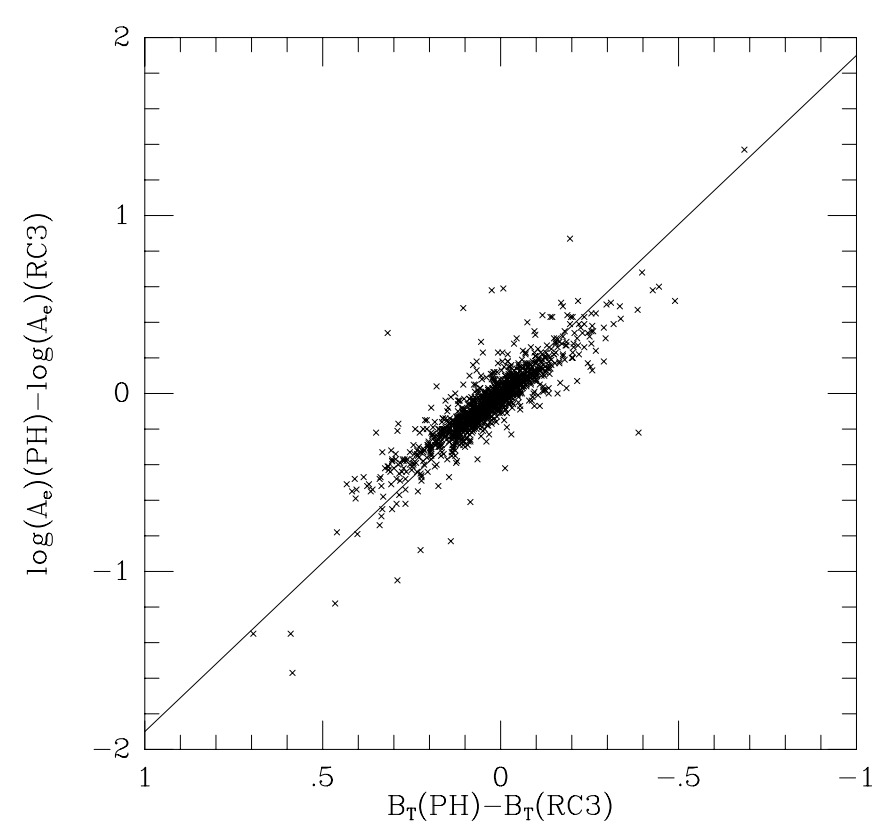

Fig. 8. Correlation of the errors on $B_{\mathrm{T}}$ and $\log \left(A_{\mathrm{e}}\right)$. Abscissa: Difference between the total magnitude $\left(B_{\mathrm{T}}\right)$ from INTERP growth curves and $B_{\mathrm{T}}$ from RC3 (in mag). Ordinate: Difference between the mean surface brightness within $A_{\mathrm{e}}$ from INTERP growth curves and RC3 in mag/arcsec ${ }^{2}$. The comparison is done for 1389 galaxies having the quality flag: $Q \leq 2$ 


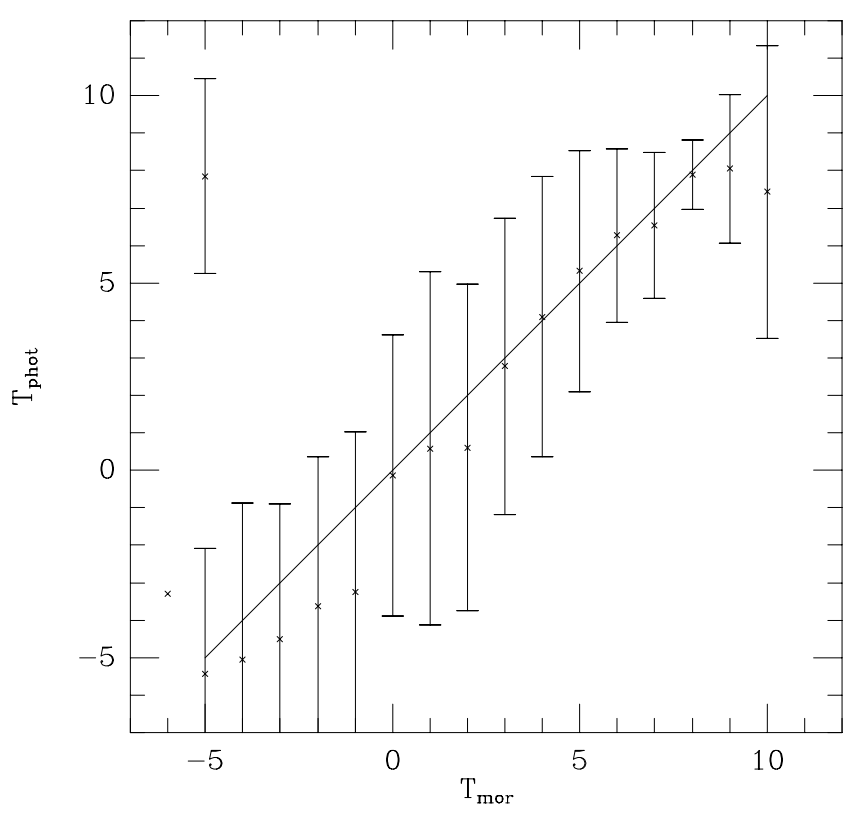

Fig. 9. Comparison between $T_{\text {mor }}$ and $T_{\text {phot }}$. $T_{\text {phot }}$ is computed with INTERP growth curves. $T_{\text {mor }}$ is from RC3. Each point is the mean $T_{\text {phot }}$ associated with a given $T_{\text {mor }}$. The error bar is the $1-\sigma$ dispersion around this value. The galaxies with $T_{\text {mor }}=-5$ and $T_{\text {phot }}>2$ have been isolated. The comparison is done for 1389 galaxies having the quality flag: $Q \leq 2$

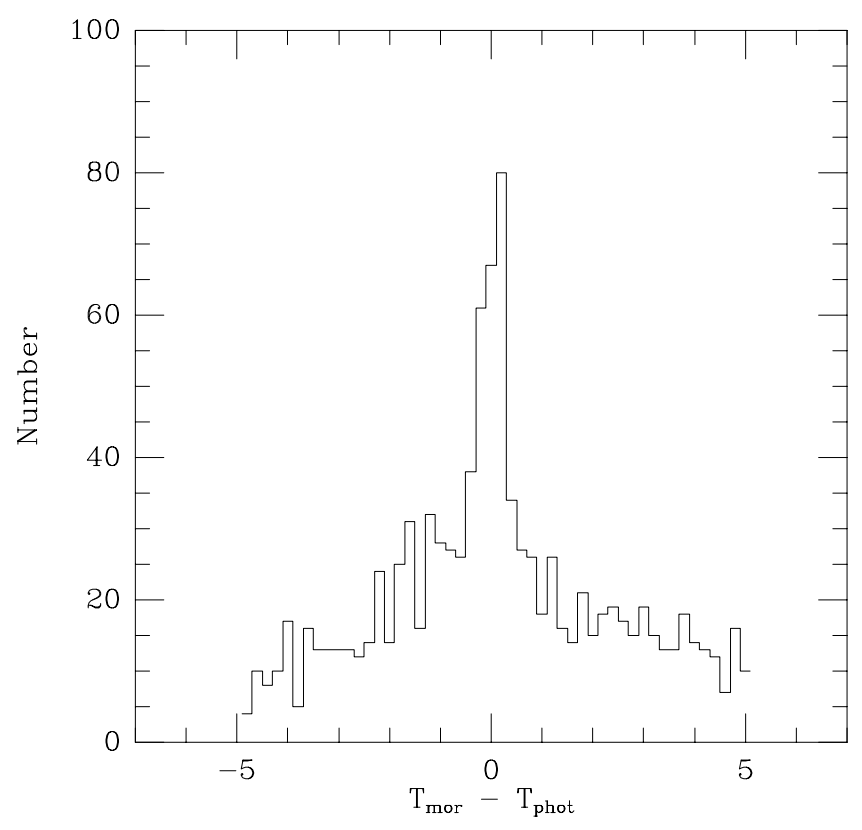

Fig. 10. Histogram of $T_{\text {mor }}-T_{\text {phot }}$
Using a one-parameter family of growth curves can probably not represent the diversity of the galaxies, and even for well observed galaxies we do not expect to reach a precision on $B_{\mathrm{T}}$ better than $0.1 \mathrm{mag}$.

\section{Comment on the morphological and photomet- ric classifications}

The morphological types served as initial guesses in our growth curves fits, but we produced photometric types which we may consider independent from morphological types.

The correlations between $T_{\text {mor }}$ and $T_{\text {phot }}$ is nevertheless very good, as seen in Fig. 9. The mean relation does not significantly depart from $T_{\text {mor }}=T_{\text {phot }}$ in the range $0 \leq T_{\text {mor }} \leq 9$. For early type galaxies, $T_{\text {phot }}<T_{\text {mor }}$, reflecting the weak dependence of the structure of these galaxies on their morphological classification. The irregular galaxies $T_{\text {mor }}=10$ have a large dispersion of $T_{\text {phot }}$. The dispersion of $T_{\text {mor }}$ at a given $T_{\text {phot }}$ is around 4 (see Fig. 10), significantly larger than the estimated error on $T_{\text {mor }}$ (around 1 in the LEDA database), and also larger than the internal errors on $T_{\text {phot }}$ (typically 0.3 ).

In Fig. 9, we have isolated the galaxies with $T_{\text {mor }}=-5$ and $T_{\text {phot }}>2$ which are likely diffuse ellipticals, characterized by an (almost) exponential profile and nevertheless classified as $T_{\text {mor }}=-5$. Indeed, the revised morphological type (de Vaucouleurs 1974, modified in RC2) includes in the same class the diffuse ( $d E$; dwarf ellipticals) and normal ellipticals $(E)$. From a physical viewpoint, however, $E$ and $d E$ are distinct families resulting probably from different evolutionnary and formation processes (Fergusson \& Binggeli 1994). Even, if a transition exists between both families, such intermediate objects are rare (Prugniel 1994).

Hence, at least for early-type galaxies, the correlation between $T_{\text {phot }}$ and $T_{\text {mor }}$ is mostly an effect of the selection of the sample. Using a volume-limited complete sample, instead of the present sample of bright galaxies, we would have found $\left\langle T_{\text {phot }}>\approx 10\right.$ for the elliptical galaxies, because the diffuse ellipticals would dominate the sample (as they do the the virgo cluster catalogue, Binggeli et al. 1984).

Finally, the photometric type characterizes the structure of galaxies, and should be considered as a new parameter, beside the morphological type.

\section{Conclusion}

In this paper, we have presented a catalogue of both photoelectric and surface photometry concerning 7744 galaxies. We explored different sets of growth curves to fit these data, and finally adopted interpolations between the de Vaucouleurs $\left(r^{1 / 4}\right)$ and exponential laws. We then derived the photometric parameters for 5169 galaxies, twice the RC3 sample. 
While the use of growth curve fitting ignores the details of the structure of galaxies: dust, arms, active nuclei... it is still the most effective method to derive homogeneously photometric parameters. The total magnitude, effective aperture and photometric type hence determined, provide a realistic basic description of the galaxies. Going further requires accurate 2-D photometric analysis (Prugniel \& D’Onofrio 1997; Héraudeau \& Simien, in preparation).

The data presented in this paper may also be conveniently consulted via Hypercat to: (http://www-obs.univlyon1.fr $/ \sim$ prugniel/cgi-bin/hypercat/).

Acknowledgements. We are grateful to R. Buta as a referee for his helpful comments on the manuscript and for sending us the revUBV catalogue. We thank D. Burstein, I. Jørgensen, G. Longo, J. Lucey, R. Peletier and P. Poulain who sent their published data in computer readable form, and C. Petit and F. Simien who helped to digitize some tables. We have made use of the Lyon-Meudon Extragalactic Database (LEDA) supplied by the LEDA team at the CRAL-Observatoire de Lyon (France) and of SIMBAD (Observatoire de Strasbourg, France)

\section{References}

Binggeli B., Sandage A., Tammann G., 1985, AJ 90, 1759

Buta R., Williams K.L., 1995, AJ 109, 543

Buta R., Corwin H.G., de Vaucouleurs G., de Vaucouleurs A., Longo G., 1995, AJ 109, 517

Caon N., Capaccioli M., Rampazzo R., 1990, A\&AS 86, 429

Dressler A., Lynden-Bell D., Burstein D., et al., 1987, ApJ 313, 42

de Vaucouleurs G., 1974, in The Formation and Dynamics of Galaxies, IAU Symp. 58, 1

de Vaucouleurs G., 1977, ApJS 33, 211 de Vaucouleurs G., de Vaucouleurs A., 1964, Reference catalog of bright galaxies, University of Texas Monographs in Astronomy No. 1 (RC1)

de Vaucouleurs G., de Vaucouleurs A., Corwin H.G., 1976, Second Reference Catalogue of Bright Galaxies, University of Texas Monographs in Astronomy No. 2 (RC2)

de Vaucouleurs G., de Vaucouleurs A., Corwin H.G., et al., 1991, Third Reference Catalogue of Bright Galaxies. Springer, New York (RC3)

de Vaucouleurs A., Longo G., 1988, Univ. Texas Monographs in Astronomy, No. 5

Djorgovski S., Davis M., 1987, ApJ 313, 59

Djorgovski S., 1985, PhD thesis, Univ. of California, Berkeley

Hamabe M. Kormendy J., 1987, in Structure and Dynamics of elliptical galaxies, IAU Symposium 127, 379

Lauberts A., Valentjin E., 1989, The Surface Photometry Catalogue of the ESO-Uppsala Galaxies (ESO-LV)

Longo G., de Vaucouleurs A., 1983, Univ. Texas Monographs in Astronomy, No. 3

Longo G., de Vaucouleurs A., 1985, Univ. Texas Monographs in Astronomy, No. 3A

Mathewson D.S., Ford V.L., Buchhorn M., 1992, ApJS 81, 413

Naim A., Lahav O., Buta R.J., et al., 1995, MNRAS, 274, 1107

Prugniel Ph., 1987, CDS Bull. 33, 17

Prugniel Ph., 1994, in Dwarf galaxies, Meylan G. \& Prugniel Ph. (eds.) ESO Proc. 49, 171

Prugniel Ph., Bica E., Klotz A., Alloin D., 1993, A\&A 98229

Prugniel Ph., D'Onofrio, M., 1997, in "Dark and visible matter in galaxies", Persic M. \& Salucci P. (eds.) ASPCS 117, 568

Prugniel, Ph., Simien F., 1994, A\&A 281, L1

Prugniel Ph., Simien F., 1995, ASPCS 86, 151

Prugniel Ph., Simien F., 1996, A\&A 309, 749

Prugniel Ph., Simien F., 1997, A\&A 321, 111

Whitford A.E., 1936, ApJ 83, 424 